\title{
Adaptabilidade de genótipos de amendoim IAC às condições do cerrado mato-grossense
}

Submetido - 10 jul. $2021 \quad$ Aprovado - 31 jul. $2021 \quad$ Publicado - 30 set. 2021

http://dx.doi.org/10.52755/sas.v.2i(edesp2)138

\begin{abstract}
Professor do Instituto Federal de Educação, Ciência e Tecnologia de Mato Grosso - IFMT, Sorriso, MT. E-mail: dacio.olibone@srs.ifmt.edu.br.
\end{abstract}

Marianitha Mariano Silva Duarte (i)

Discente de Engenharia Agronômica, Instituto Federal de Educação, Ciência e Tecnologia de Mato Grosso - IFMT, Sorriso, MT. E-mail: marianithamariano@gmail.com.

Pesquisador Científico do IAC. E-mail: ignacio.godoy@sp.gov.br. Ignácio José de Godoy iD Ana Paula Encide Olibone (D)

Professora do Instituto Federal de Educação, Ciência e Tecnologia de Mato Grosso - IFMT, Sorriso, MT. E-mail: ana.olibone@srs.ifmt.edu.br.

Sergio Soares Filho iD

Discente de Engenharia Agronômica, Instituto Federal de Educação, Ciência e Tecnologia de Mato Grosso - IFMT, Sorriso, MT. E-mail: sergios7soares@gmail.com.

Engenheiro Agrônomo, Beatrice Comércio Importação e Exportação de Amendoim Ltda. Tupã-SP. E-mail: rodolfopiresribeiro@gmail.com.

\section{RESUMO}

O amendoim que anteriormente ficava restrito às áreas de renovação de canavial no estado de São Paulo, principal produtor, hoje abrange outro cenário despertando 0 interesse de produtores de outras regiões. Assim é necessário a avaliação de genótipos do Programa de Melhoramento do IAC às condições do Cerrado Mato-Grossense. Neste trabalho, a adaptabilidade e produtividade de amendoim em vagens nas condições de Sorriso-MT, foram avaliadas. Para isso, o experimento foi conduzido em blocos inteiramente casualizados, com 11 genótipos desenvolvidos pelo IAC (as cultivares IAC 503, IAC 505, IAC OL3, IAC OL5, IAC OL6, IAC Sempre Verde e as linhagens IAC 1120, IAC 1127, IAC 1129, IAC 10719 e IAC 10677) e quatro repetições. As parcelas foram compostas por quatro linhas espaçadas $0,90 \mathrm{~m}$ entre si e $4 \mathrm{~m}$ de comprimento. Aos 138 dias após a semeadura, antes da colheita foi avaliado a severidade de mancha preta (Cercosporidium personatum). Posteriormente, as plantas da área útil foram colhidas manualmente para determinação da produtividade de vagens, rendimento de grãos e massa de 100 grãos. Os dados foram submetidos à análise de variância pelo teste $F$ e as médias dos tratamentos foram agrupadas pelo teste de Scott-Knott a $5 \%$ de probabilidade. Os genótipos testados não apresentaram diferença de produtividade, mas destaca-se a produtividade do genótipo 10667 tendo potencial como nova cultivar. A massa de 100 grãos, rendimento de grãos e severidade de $C$. personatum apresentou diferença entre os genótipos. Nas condições experimentais, todos os genótipos apresentaram ótima adaptabilidade produtiva e moderada incidência de doença e a cultivar IAC Sempre Verde não apresentou incidência de mancha preta.

Palavras-chave: Arachis hypogaea L.; Produtividade; Cercosporidium personatum.

\section{Adaptability of IAC peanut genotypes to Mato Grosso Cerrado conditions}

Este é um trabalho de acesso aberto e distribuído sob os Termos da Creative Commons Attribution Attribution-NonCommercial-ShareAlike 4.0 International. 


\begin{abstract}
Peanuts, which were previously restricted to sugarcane renovation areas in the state of São Paulo, the main producer, now encompasses another scenario, arousing the interest of producers from other regions. Thus, it is necessary to evaluate genotypes from the IAC Breeding Program under the conditions of the Cerrado Mato-Grossense. In this work, the adaptability and productivity of peanuts in pods under the conditions of Sorriso-MT were evaluated. For this, the experiment was carried out in completely randomized blocks, with 11 genotypes developed by the IAC (the cultivars IAC 503, IAC 505, IAC OL3, IAC OL5, IAC OL6, IAC Semper Verde and the lines IAC 1120, IAC 1127, IAC 1129, IAC 10719 and IAC 10677) and four repetitions. The plots were composed of four lines spaced $0.90 \mathrm{~m}$ apart and $4 \mathrm{~m}$ long. At 138 days after sowing, before harvesting, the severity of black spot (Cercosporidium personatum) was evaluated. Subsequently, the plants in the useful area were manually harvested to determine the pod yield, grain yield and mass of 100 grains. Data were subjected to analysis of variance using the $F$ test and the treatment means were grouped using the ScottKnott test at $5 \%$ probability. The tested genotypes showed no difference in productivity, but the productivity of genotype 10667 stands out, having potential as a new cultivar. The 100-grain mass, grain yield and severity of $C$. personatum differed between genotypes. Under the experimental conditions, all genotypes showed excellent productive adaptability and moderate incidence of disease and the cultivar IAC Semper Verde showed no incidence of black spot.
\end{abstract}

Keywords: Arachis hypogaea L.; Yield; Cercosporidium personatum.

\title{
Adaptabilidad de los genotipos de maní IAC a las condiciones del Cerrado de Mato Grosso
}

\begin{abstract}
RESUMEN
El maní, que antes estaba restringido a las áreas de renovación de caña de azúcar en el estado de São Paulo, el principal productor, ahora abarca otro escenario, despertando el interés de productores de otras regiones. Por lo tanto, es necesario evaluar los genotipos del Programa de Mejoramiento del IAC en las condiciones del Cerrado Mato-Grossense. En este trabajo se evaluó la adaptabilidad y productividad del maní en vaina bajo las condiciones de Sorriso-MT. Para ello, el experimento se realizó en bloques completamente al azar, con 11 genotipos desarrollados por el IAC (los cultivares IAC 503, IAC 505, IAC OL3, IAC OL5, IAC OL6, IAC Semper Verde y las líneas IAC 1120, IAC 1127, IAC 1129, IAC 10719 e IAC 10677) y cuatro repeticiones. Las parcelas estaban compuestas por cuatro líneas espaciadas $0.90 \mathrm{~m}$ de distancia y $4 \mathrm{~m}$ de largo. A los 138 días después de la siembra, antes de la cosecha, se evaluó la severidad de la mancha negra (Cercosporidium personatum). Posteriormente, las plantas del área útil se cosecharon manualmente para determinar el rendimiento de vaina, rendimiento de grano y peso de 100 granos. Los datos se sometieron a análisis de varianza mediante la prueba F y las medias de los tratamientos se agruparon mediante la prueba de Scott-Knott al $5 \%$ de probabilidad. Los genotipos probados no mostraron diferencia en productividad, pero se destaca la productividad del genotipo 10667, que tiene potencial como nuevo cultivar. La masa de 100 granos, el rendimiento de grano y la gravedad de $C$. personatum difirieron entre los genotipos. En condiciones experimentales, todos los genotipos mostraron excelente adaptabilidad productiva y moderada incidencia de enfermedades y el cultivar IAC Semper Verde no mostró incidencia de mancha negra.
\end{abstract}

Palabras clave: Arachis hypogaea L.; Productividad; Cercosporidium personatum.

\section{Introdução}

A cultura do amendoim (Arachis hypogaea L.) que anteriormente ficava restrita às áreas de renovação de canavial no estado de São Paulo, principal produtor, hoje abrange outro cenário despertando o interesse de 
produtores de outras regiões. Em Mato Grosso, tem atraído produtores da região médio norte, onde nas últimas duas safras apresentou um crescimento de $624 \%$ na área cultivada. Dados experimentais obtidos no município de Sorriso-MT, desde a safra 2018/19 têm demostrado que a cultura tem boa adaptação as condições ambientais da região com ótimas produtividades em plantios realizados entre novembro e janeiro (OLIBONE et al., 2020).

A região compreendida pelo bioma Cerrado tem sido a região de expansão da produção para as principais commodities brasileiras e pode ser uma região de expansão da cultura do amendoim no Brasil (SUASSUNA et al., 2018).

O cultivo dessa leguminosa é uma alternativa viável para o Cerrado, pois se adapta muito bem aos solos de textura média, bem drenados, soltos e friáveis, condições comuns nesse bioma. O solo é o meio onde se desenvolvem não somente as raízes, mas também os frutos, em função disso a aeração e a boa drenagem do solo são de fundamental importância para a cultura, pois solos de textura argilosa, pesados, dificultam a penetração do ginóforo e provocam problemas na colheita (NOGUEIRA; TÁVORA, 2005).

Aconselha-se cultivo em solos de textura arenosa a franco arenosa. Quando o solo apresenta problemas de drenagem, dificulta o crescimento dos frutos e a penetração do ginóforo. Outro problema com solos pesados é a dificuldade de colher os frutos; dessa forma, solos arenosos são recomendados para cultivares com frutos grandes, evitando maiores perdas mecânicas durante a colheita (NOGUEIRA; TÁVORA, 2005).

Para a Secretaria de Desenvolvimento Econômico (SEDEC, 2020) à aptidão agrícola, as terras favoráveis, o uso de tecnologias, o clima definido, os programas de incentivos, o empenho de pesquisadores, a avidez dos produtores, a mão-de-obra qualificada, o comprometimento dos profissionais da área rural, formam o conjunto de ferramentas para o desenvolvimento de alternativas econômicas para Mato Grosso com potencial para produzir e industrializar o amendoim. De acordo com o 
Relatório de Produção de Grãos do Estado de Mato Grosso, fornecido pela Sedec (2020) os maiores incrementos na produção ocorreram no Amendoim $1^{\text {a }}$ safra $(72 \%)$. Assim, observamos que a cultura vem ganhando espaço.

No entanto, essa expansão poderá levar ao aumento dos problemas fitossanitários da cultura, especialmente no que se refere às doenças. Dessa forma, isso exige um preparo e avaliação do potencial de dano que as doenças podem representar à cultura e assim alternativas que as minimizem. As doenças foliares são o principal gargalo para a produção dessa cultura e de acordo com Moraes e Godoy (1997), nas condições brasileiras a principal é a mancha preta (Cercosporidium personatum).

Portanto, avaliar o comportamento de diferentes genótipos à essas condições ambientais são importantes para o desenvolvimento de uma cadeia produtiva do amendoim com sustentabilidade e obstante, a avaliação de materiais genéticos desenvolvidos pelo Programa de Melhoramento do amendoim do IAC devem fazer parte desse processo de crescimento.

Nesse contexto, o presente trabalho foi desenvolvido com o objetivo de avaliar a adaptabilidade e a incidência de mancha preta em diferentes genótipos de amendoim às condições de Cerrado Mato-Grossense.

\section{Materiais métodos}

O experimento foi desenvolvido no ano agrícola 2020/2021, na Fazenda experimental do Instituto Federal de Educação, Ciência e Tecnologia de Mato Grosso - Campus Sorriso, localizado no município de Sorriso-MT, cujas coordenadas geográficas são $55^{\circ} 48^{\prime} 07^{\prime \prime}$ W e $12^{\circ} 41^{\prime} 43^{\prime \prime}$ $\mathrm{S}$, com altitude média de $358 \mathrm{~m}$. O solo é classificado como Latossolo Vermelho Amarelo distrófico com textura média. O clima da região é tipo Aw (KÖPPEN; GEIGER, 1928), com temperatura média de $26,2^{\circ} \mathrm{C}$ e pluviosidade média de $1970 \mathrm{~mm}$ anuais os quais são distribuídos nos meses de outubro a abril (SOUZA et al., 2013). 
O delineamento experimental utilizado foi em blocos inteiramente casualizados, com 11 genótipos desenvolvidos pelo Programa de Melhoramento de Amendoim do Instituto Agronômico de Campinas - IAC, sendo as cultivares IAC 503, IAC 505, IAC OL3, IAC OL5, IAC OL6 e IAC Sempre Verde e as linhagens IAC 1120, IAC 1127, IAC 1129, IAC 10719 e IAC 10677, com quatro repetições. De acordo com Godoy et al. (2018), as cultivares e linhagens apresentam as seguintes características:

- IAC 503 apresenta ciclo de 140-150 dias com potencial produtivo de $6.500 \mathrm{~kg} \mathrm{ha}^{-1}$, plantas tolerantes a doenças foliares, a virose e estiagem. Grãos de formato oblongo, calibre predominante $38 / 42$ e química alto oleico.

- IAC 505 apresenta ciclo de 130-140 dias e potencial produtivo de $6.500 \mathrm{~kg} \mathrm{ha}^{-1}$; plantas tolerantes a doenças foliares, a virose e estiagem. Grãos arredondados, calibre predominante 40/50 e química alto oleico.

- IAC OL3 apresenta ciclo de 125-130 dias e alto potencial produtivo, aproximadamente $7.000 \mathrm{~kg} \mathrm{ha}^{-1} \mathrm{com}$ as doenças foliares totalmente controladas, possui a química alto oleico e grãos arredondados com alta proporção de calibre 38/42.

- IAC OL5 apresenta ciclo de 130-135 dias e potencial produtivo de $6.000 \mathrm{~kg} \mathrm{ha}^{-1}$, plantas rústicas e tolerantes a doenças foliares e a estiagem, de grãos arredondados, calibre predominante 40/50 e química alto oleico.

- IAC OL6 com ciclo de 130-140 dias e potencial produtivo de 6.500 $\mathrm{kg} \mathrm{ha}^{-1}$, plantas tolerantes a doenças foliares e grãos arredondados, calibre predominante $38 / 42$ e química alto oleico.

- IAC Sempre Verde com ciclo de 130-140 dias, potencial produtivo $5.000 \mathrm{~kg} \mathrm{ha}^{-1}$, altamente resistente à mancha preta e ferrugem, grãos arredondados de pele vermelha, calibre 60/70, aptidão para cultivo orgânico devido à resistência a doenças.

- IAC 1120, 1127 e 1129 são linhagens alto oleicas selecionadas para ciclo de 125-130 dias nas condições de SP. 
- IAC 10677 e 10719 são linhagens com ciclo de 130-135 dias, associam alta produtividade com rusticidade, grãos alto oleicos, bem padronizados para exportação tipo Runner.

As parcelas foram compostas por quatro linhas espaçadas $0,90 \mathrm{~m}$ entre elas com $4 \mathrm{~m}$ de comprimento (14,4 $\mathrm{m}^{2}$ por parcela), considerando-se as duas linhas de cada extremidade como bordadura e duas linhas centrais como área útil $\left(3,6 \mathrm{~m}^{2}\right)$, desprezando-se $1,0 \mathrm{~m}$ de cada extremidade.

Antecedendo a implantação do experimento, foi efetuado o levantamento da fertilidade e granulometria do solo, utilizando-se metodologia proposta por Raij et al. (2001) e Embrapa (1997), na profundidade de 0 a $0,20 \mathrm{~m}$. Os resultados das análises foram: $\mathrm{pH}\left(\mathrm{CaCl}_{2}\right)$

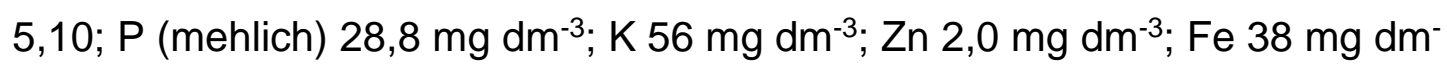

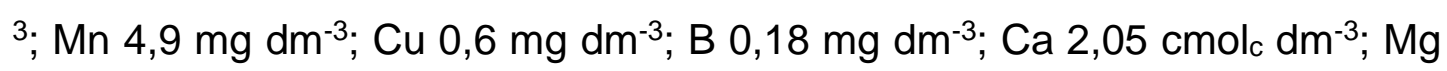
$0,65 \mathrm{cmol}_{\mathrm{c}} \mathrm{dm}^{-3}$; Al 0,0 $\mathrm{cmol}_{\mathrm{c}} \mathrm{dm}^{-3} \mathrm{e} \mathrm{H}+\mathrm{Al} 3,5 \mathrm{cmol}_{\mathrm{c}} \mathrm{dm}^{-3}$ com saturação por bases $45,1 \%$ e matéria orgânica 1,99\%. A análise granulométrica de areia, silte e argila apresentou 682, 56 e $262 \mathrm{~g} \mathrm{dm}^{-3}$, respectivamente.

A adubação foi realizada com a aplicação a lanço em área total 500 $\mathrm{kg} \mathrm{ha}^{-1}$ do formulado 00-18-18 (90 kg ha-1 de $\mathrm{P}_{2} \mathrm{O}_{5}$ e $90 \mathrm{~kg} \mathrm{ha}^{-1}$ de $\mathrm{K}_{2} \mathrm{O}$ ) e em seguida o solo foi preparado com 2 gradagens pesada e 1 gradagem com niveladora e em seguida, em 10 de dezembro de 2020, foi realizada a semeadura manual do amendoim, distribuindo-se 25 sementes por metro linear (germinação 90\%). Em cobertura, aos 35 dias após a emergência (DAE), foi realizada a aplicação de $500 \mathrm{~kg} \mathrm{ha}^{-1}$ de gesso agrícola, aplicado a lanço em área total.

O manejo fitossanitário da área experimental foi realizado com aplicações em 09/01 (chlorothalonil + indoxacarb + novalurom + acetamiprid + pyriproxyfen), em 05/02 (chlorothalonil + trifloxystrobin + cyproconazole), em 21/02 chlorothalonil + imidacloprid + bifenthrin + indoxacarb + novalurom), em 04/03 (chlorothalonil I + trifloxystrobin + cyproconazole + imidacloprid + bifenthrin + indoxacarb + novalurom), em 11/03 (chlorothalonil + trifloxystrobin + cyproconazole + indoxacarb + novalurom + chlorfenapyr), em 24/03 (chlorothalonil + pyraclostrobin + epoxiconazole) e em 03/04 
(chlorothalonil + pyraclostrobin + epoxiconazole + chlorfenapyr) utilizando as doses recomendadas pelos fabricantes.

A severidade de mancha preta (Cercosporidium personatum) foi avaliada usando a escala diagramática de severidade com notas de 1 a 9 ao final do ciclo dos genótipos, antes da inversão do experimento (SUBRAHMANYAM et al., 1982).

A colheita foi realizada aos 138 dias após o plantio (DAP). Foram avaliados massa de 100 grãos $(\mathrm{g})$ e produtividade de vagens $\left(\mathrm{kg} \mathrm{ha}^{-1} \mathrm{e}\right.$ sacas alqueire ${ }^{-1}$ ) mediante a pesagem de vagens e grãos, da área de 3,6 $\mathrm{m}^{2}$ centrais das duas linhas da parcela. A avaliação de rendimento (\%) consistiu na retirada de uma amostra aleatória de amendoim em vagens de cada parcela sendo considerado $100 \%$. Após o beneficiamento mecanizado simulando um sistema industrial foi feito a massa de grãos e a casca foi desprezada, com isso calculando a porcentagem de rendimento das parcelas.

Os dados obtidos foram submetidos à análise de variância (Teste F) e as médias dos tratamentos foram comparados pelo teste de Scott-Knott a $5 \%$ de probabilidade, por meio do programa computacional SISVAR 5.6 (FERREIRA, 2019).

\section{Resultados e discussão}

A análise de variância para as variáveis está apresentada na Tabela 1 , onde podemos observar que não houve diferença significativa entre os genótipos para a produtividade de amendoim em vagens. Para massa de 100 grãos, rendimento de grãos e severidade de $C$. personatum houve diferença entre os tratamentos. Na massa de 100 grãos, observa-se que 0 teste de Scott-Knott formou três grupos, onde os genótipos IAC 503, IAC 505, IAC OL3, IAC OL6, IAC 1120, IAC 1127, IAC 1129, IAC 10719 e IAC 10677 apresentaram a maior média, resultando na maior granulometria (Tabela 2). O genótipo IAC OL5 apresentou M100 intermediária, com 52,53 g em 100 sementes e o genótipo IAC Sempre Verde apresentou a menor M100, com 46,18 g. 
Tabela 1. Resumo da análise de variância para massa de 100 grãos (g) (M100), rendimento de grãos (REND), produtividade de vagens $\left(\mathrm{kg} \mathrm{ha}^{-1}\right) \mathrm{e}$ (sc alq $^{-1}$ ) (PROD) e severidade de mancha preta (Cercosporidium personatum) (nota) em função dos genótipos de amendoim. Sorriso-MT, 2020/21.

\begin{tabular}{lcccc}
\hline \multirow{2}{*}{$\begin{array}{c}\text { Fontes de } \\
\text { variação }\end{array}$} & \multicolumn{4}{c}{ Quadrados Médios } \\
\cline { 2 - 5 } & M100 $\mathbf{( g )}$ & REND (\%) & PROD $\left(\mathbf{k g ~ h a}^{-1}\right)$ & Severidade $^{*}$ \\
\hline Genótipos & $65,0^{*}$ & $46,3^{* *}$ & $731600,6^{\text {ns }}$ & $11,1^{* *}$ \\
Rep & $51,4^{\text {ns }}$ & $9,4^{*}$ & $3506352,1^{*}$ & $21,0^{* *}$ \\
erro & 18,0 & 2,3 & 723449,3 & 1,5 \\
\hline CV (\%) & 7,49 & 1,88 & 15,92 & 31,82 \\
\hline ns não significativo; ${ }^{*} e^{* *}$ significativo a 5 e $1 \%$ de probabilidade pelo teste Scott-Knott, \\
respectivamente.
\end{tabular}

Tabela 2. Massa de 100 grãos (g), rendimento (\%) e produtividade de vagens ( $\mathrm{kg} \mathrm{ha}^{-1}$ e sacas alqueire ${ }^{-1}$ ) e severidade de mancha preta (Cercosporidium personatum) (Nota) em função de diferentes genótipos de amendoim. Sorriso-MT, 2020/21.

\begin{tabular}{|c|c|c|c|c|c|}
\hline \multirow{2}{*}{ Genótipos } & \multicolumn{5}{|c|}{ Médias } \\
\hline & M100 (g) & \% Rend. & Prod. (kg ha- & Prod. (sc alq-1) & Severidade \\
\hline IAC 503 & $58,43 \mathrm{a}$ & $81,89 a$ & $5563,89 a$ & 539 & $3,00 \mathrm{~b}$ \\
\hline IAC 505 & $58,92 \mathrm{a}$ & $83,12 \mathrm{a}$ & 5649,30 a & 547 & $3,25 b$ \\
\hline IAC OL3 & $57,23 \mathrm{a}$ & $83,08 \mathrm{a}$ & $5280,55 \mathrm{a}$ & 511 & $6,50 \mathrm{a}$ \\
\hline IAC OL5 & $52,53 b$ & $81,46 a$ & $5724,30 a$ & 554 & $2,75 b$ \\
\hline IAC OL6 & $59,73 a$ & $80,88 a$ & $5134,72 \mathrm{a}$ & 497 & $2,75 b$ \\
\hline IAC 1120 & $59,64 \mathrm{a}$ & $80,33 \mathrm{a}$ & $5391,66 a$ & 522 & $4,00 \mathrm{~b}$ \\
\hline IAC 1127 & $56,89 a$ & $81,65 \mathrm{a}$ & $5319,44 a$ & 515 & $4,75 a$ \\
\hline IAC 1129 & $57,46 \mathrm{a}$ & $82,43 \mathrm{a}$ & $4577,77 \mathrm{a}$ & 443 & $6,25 \mathrm{a}$ \\
\hline IAC 10719 & $56,64 \mathrm{a}$ & $81,66 \mathrm{a}$ & $5149,30 a$ & 498 & $5,55 a$ \\
\hline IAC 10677 & $59,53 \mathrm{a}$ & $80,73 \mathrm{a}$ & $6129,86 a$ & 593 & $3,75 b$ \\
\hline IAC S. Verde & $46,18 \mathrm{C}$ & $70,82 \mathrm{~b}$ & $4844,44 a$ & 469 & $1,00 \mathrm{~b}$ \\
\hline
\end{tabular}

Letras iguais na coluna pertencem ao mesmo grupo, de acordo com o teste Scott-Knott a $5 \%$ de probabilidade.

Quanto ao rendimento de grãos, houve diferença significativa entre o genótipo IAC Sempre Verde e os demais, onde este apresentou rendimento 
médio de 70,82 \%. O menor rendimento de grãos do IAC Sempre Verde se deve a menor M100 grãos (46,18 g), o que pode estar relacionado com a maior espessura da casca em comparação aos demais genótipos.

A produtividade de amendoim em vagens entre os genótipos não foi diferente. Contudo, destaca-se o genótipo IAC 10677 com produtividade de $6.129,8 \mathrm{~kg} \mathrm{ha}^{-1}\left(593,4 \mathrm{sc} \mathrm{alq}^{-1}\right)$ indicando que está linhagens tem grande potencial para torna-se uma nova cultivar, pois além de potencial produtivo, também apresentou mediana incidência de mancha preta (Tabela 2).

A produtividade média do experimento foi de $5.342,3 \mathrm{~kg} \mathrm{ha}^{-1}$ (517 sc $\mathrm{alq}^{-1}$ ), sendo aproximadamente $45 \%$ superior à média nacional divulgada pela CONAB (2021) de amendoim primeira safra $\left(3.679,0 \mathrm{~kg} \mathrm{ha}^{-1}\right)$. Vale destacar que a cultivar IAC Sempre Verde, mesmo apresentando a menor produtividade absoluta neste experimento, foi superior à média nacional. Portanto, pode ser uma ótima opção de cultivo em sistemas agroecológicos, uma vez que não há necessidade de uso de fungicidas durante o ciclo pela ausência da doença.

Para a avaliação da severidade de mancha preta (Cercosporidium personatum) o genótipo IAC Sempre Verde apresentou nota mínima $(1,0)$, demostrando que este genótipo possui alta resistência genética a doença. Os genótipos IAC OL3 (nota 6,5), IAC 1127 (nota 4,75), IAC 1129 (nota 6,25) e IAC 10719 (nota 5,55) formaram o grupo com a maior severidade da doença. Rizzi et al. (2019) e Rizzi et al. (2020) avaliando as cultivares BRS 421 OL e BRS 423 OL obtiveram severidade com notas 5,9 e 5,7 respectivamente, nas condições de Sorriso-MT.

\section{Conclusões}

Para as condições ambientais em que foi conduzido o experimento, todos os genótipos apresentaram ótima adaptabilidade produtiva e moderada incidência de doença e a cultivar IAC Sempre Verde não apresentou incidência de mancha preta possibilitando o cultivo em sistemas agroecológicos. 


\section{Agradecimentos}

Os autores agradecem aos discentes do curso de Engenharia Agronômica do IFMT Campus Sorriso membros do grupo de pesquisa Sistemas de Produção Agrícola no Cerrado que contribuíram na condução do experimento. Ao Programa de Melhoramento do Amendoim - IAC, a LC Sementes, a Fazenda São José, a MIAC - Indústrias Colombo e a Beatrice Peanuts. 


\section{Referências}

CONAB. Acompanhamento da Safra Brasileira de Grãos. Safra 2020/21 Nono levantamento, v. 8, n. 9, p. 1-121, 2021. Disponível em: <https://www.conab.gov.br/info-agro/safras>. Acesso em: 27 jun. 2021.

EMBRAPA. Centro Nacional de Pesquisa de Solos (Rio de Janeiro, RJ). Manual de métodos de análise de solo. 2.ed. Rio de Janeiro Embrapa CNPS, 1997. 212p.

FERREIRA, D. F. SISVAR: um sistema de análise de computador para efeitos fixos projetos de tipo de partida dividida. Revista Brasileira de Biometria, v. 37, n. 4, p. 529-535, 2019.

GODOY, I. J.; SANTOS, J.F.; MORAES, A.R.A.; CARVALHO, C.L.; BOLONHEZI, D.; MICHELOTTO, M.D.; FINOTO, E.L.; MARTINS, A.L.M.; FREITAS, R.; CAVICHIOLI, J.C. Cultivares de amendoim alto oleicos: uma inovação para o mercado produtor e consumidor brasileiros. O Agronômico, Boletim Técnico-Informativo do Instituto Agronômico, 2018.

KÖPPEN, W.; GEIGER, R. Klimate der Erde. Gotha: Verlag condicionadas. Justus Perthes. 1928.

MORAES, S.A.; GODOY, I.J. Amendoim - Controle de Doenças. In: ZAMBOLIM, L. \& VALE, F.X.R. (eds.) Controle de doenças de plantas: Grandes culturas. Viçosa, Universidade Federal de Viçosa; Brasília, Ministério da Agricultura e do Abastecimento, Suprema Gráfica e Editora Ltda., 1:1- 49, 1997.

NOGUEIRA, R. J. M. C.; TÁVORA, F. J. A. F. Ecofisiologia do amendoim (Arachis hypogaea L.) In: SANTOS, R. C. $\mathbf{O}$ agronegócio do amendoim no Brasil. Campina grande: Embrapa - CNPA, 2005, 451 p.

OLIBONE, D.; WERNER, F. P.; HEUERT, J.; PIVETTA, L. G.; DUARTE, M. M. S.; XAVIER, M. F. N. Desempenho agronômico de cultivares de amendoim em diferentes épocas de semeadura nas condições de SorrisoMT. South American Sciences, v. 1, n. 2, p. e2041, 2020.

RAIJ, B.; ANDRADE, J. C.; CANTARELLA, H.; QUAGGIO, J. A. Análise química para avaliação da fertilidade de solos tropicais. Campinas Instituto Agronômico, 2001. 285p.

RIZZI, T. S.; OLIBONE, D.; HEUERT, J.; XAVIER, M. F. N.; MOURA, G. M.; SUASSUNA, T. M. F. Desempenho agronômico de genótipos de amendoim nas condições de Sorriso-MT. South American Sciences, v. 1, n. 2, p. e2043, 2020.

RIZZI, T. S.; OLIBONE, D.; LODEA, L.; HEUERT, J.; XAVIER, M. F. N.; SUASSUNA, T. M. F. Desempenho de cultivares de amendoim na região médio-norte Mato-grossense. In: Anais do encontro sobre a cultura do 
amendoim, 16., 2019, Jaboticabal. Anais eletrônicos... Campinas, GALOÁ, 2019.

SEDEC. Potencial produtivo do amendoim é realidade para o estado de Mato Grosso. 2020. Disponível em: <https://www.noticiasagricolas.com.br/noticias/agronegocio/267880potencial-produtivo-do-amendoim-e-realidade-para-o-estado-de-matogrosso.html\#.YOCub-hKjlU>. Acesso em: 30 jun. 2021.

SOUZA, A. P.; MOTA, L. L.; ZAMADEI, T. MARTIM, C. C.; ALMEIDA, F. T.; PAULINO, J. Classificação climática e balanço hídrico climatológico no estado de Mato Grosso. Nativa, v. 1, n. 1, p. 34-43, 2013.

SUASSUNA, T. M; HEUERT, J; BOGGIANI, J. C; PERINA, F. J; SOFIATTI, V; BETTINI, P. C; OLIVEIRA, M. C. T; LEONEL, C. L. Desempenho de linhagens de amendoim na região do Cerrado In: Encontro sobre a cultura do amendoim, 2018, Jaboticabal. Anais. Jaboticabal: Unesp, 2018.

SUBRAHMANYAM, P.; MCDONALD, D.; GIBBONS, R. W.; NIGAM, S. N.; NEVILL, D. J. Resistance to rust and late leaf spot diseases in some genotypes of Arachis hypogaea. Peanut Science, v. 9, p. 9-14, 1982. 